\title{
Prognostic value of lymphocyte cell ratios in peritoneal dialysis
}

\author{
Roberto C. Marques ${ }^{1}$, Eduarda C. Carias ${ }^{1}$, Ana T. Domingos ${ }^{1}$, Anabela M. Guedes ${ }^{1,2}$, Idalécio Bernardo ${ }^{1}$, Pedro L. Neves ${ }^{1,2}$ \\ ${ }^{1}$ Nephrology Department - Centro Hospitalar Universitário do Algarve, Faro, Portugal \\ 2 Department of Biomedical Sciences and Medicine - University of Algarve, Faro, Portugal
}

\section{ABSTRACT}

Background: Neutrophil-to-lymphocyte ratio (NLR) and platelet-to-lymphocyte ratio (PLR) have been introduced as useful inflammatory markers to predict the outcome of a wide spectrum of diseases, such as malignancies and cardiovascular pathologies. Limited evidence is available for their role in end-stage renal disease and dialysis patients. The aim of this study was to evaluate NLR and PLR as predictors of mortality in peritoneal dialysis (PD) patients. Methods: In this retrospective study 122 incident PD patients between 2004 and 2019 were included. Demographic, clinical and laboratory data were collected. Relationships between NLR, PLR and high-sensitivity C-reactive protein (hs-CRP) were evaluated by Spearman correlation test. Univariable and multivariable Cox regression analysis were performed to determine the association of NLR and PLR with all-cause mortality. Results: Mean levels of NLR and PLR were 3.99 \pm 2.6 and 195.5 \pm 101.7 , respectively. Both NLR and PLR were significantly and positively correlated with serum hs-CRP levels ( $r=0.340, p<0.001$ and $r=0.360, p<0.001$, respectively). The overall mortality rate was $18.9 \%$ after a mean follow-up of $30.2 \pm 24.0$ months. On multivariable modeling, we found that higher NLR $(\mathrm{HR}=1.662,95 \% \mathrm{Cl} 1.117-2.472)$ and higher PLR (HR=1.010,95\% $\mathrm{Cl} 1.004-1.015)$, in addition to lower residual renal function and higher Charlson comorbidity index were significant independent predictors of poor survival, when adjusted for nutritional status. Discussion: In this study, NLR and PLR were validated as inflammatory markers and predicted survival in our PD patients. Our results suggest that NLR might be a better indicator of mortality than PLR.

Key-words: Neutrophil-to-lymphocyte ratio, platelet-to-lymphocyte ratio, inflammation, mortality.

\section{INTRODUCTION}

Chronic inflammation plays an important role in the development and progression of various chronic conditions and diseases such as obesity, diabetes, cardiovascular disease, cancer, chronic obstructive lung disease and chronic kidney disease $(C K D)^{1-3}$. Patients with CKD tend to have elevated levels of inflammatory mediators, which accelerates the progression of atherosclerosis, the most important cause of morbidity and mortality in CKD 4 .

In addition to known conventional indicators of inflammation such as C-reactive protein (CRP), fibrinogen, erythrocyte sedimentation rate and several interleukins, studies have shown that neutrophil-to-lymphocyte ratio (NLR) and platelet-to-lymphocyte ratio (PLR), readily available biomarkers calculated from complete blood count (CBC), are also increased during inflammation and have been used as prognostic factors in cardiovascular and oncologic diseases ${ }^{5,6}$.

Recent studies have indicated that NLR and PLR offer a plausible strategy in clinical practice for evaluation of inflammation in endstage renal disease (ESRD) and may predict mortality among patients with CKD, including hemodialysis patients ${ }^{7-10}$. However, their prognostic value in PD is still unknown. The aim of this study was to evaluate NLR and PLR as predictors of all-cause mortality in PD patients.

\section{METHODS}

\section{Study population}

We retrospectively examined data from all ESRD patients aged $\geq$ 18 years who initiated PD at the Centro Hospitalar Universitário do Algarve from January 1, 2004 to April 30, 2019 and who had a peritoneal equilibration test (PET). They were treated with continuous ambulatory PD or automated PD. Patients were followed up from the first date of dialysis until death, kidney transplantation, loss to follow-up or the date of final follow-up assessment for all patients (April 30, 2019).

Clinical and biochemical data at the time of PET were recorded, including age, Charlson comorbidity index (CCI), pre-albumin as marker of nutritional status, normalized glomerular filtration rate (nGFR) and presence of diabetes mellitus. Blood samples for $\mathrm{CBC}$ and biochemistry panels were collected for each patient early in the morning before the first exchange of day and immediately analyzed in our hospital laboratory. For CBC analysis, an automatic blood counter was used. NLR was calculated using neutrophil count divided by lymphocyte count whereas PLR was calculated using platelet count divided by lymphocyte count, both obtained from the same automated blood sample. Weekly Kt/V urea was determined by adding renal Kt (24-hour urine urea nitrogen content/serum urea nitrogen) and peritoneal Kt (24-hour dialysate urea nitrogen content/serum urea nitrogen) normalized to the patient's estimated urea distribution volume (V). 


\section{Statistical analysis}

Categorical variables are presented as frequencies and percentages, and continuous parametric variables as mean and standard deviations (SD). Normal distribution was checked using skewness and kurtosis. Correlations between NLR, PLR and serum high-sensitivity C-reactive protein (hs-CRP) were evaluated using the Spearman correlation test. Adjusted survival curves were estimated using the Cox average method. A backward elimination procedure based on likelihood ratio was then carried out to remove superfluous variables from the model until the most parsimonious model was identified. Data were analyzed using the software package Statistical Package for the Social Science (SPSS) 23.0 for Windows. P-values less than 0.05 were considered significant.

\section{RESULTS}

\section{Patient characteristics}

A total of 164 incident patients started PD during the study period, but only 122 had a PET and were included. The mean age and $\mathrm{CCl}$ was $55.0 \pm 17.5$ years and $5.0 \pm 2.5$ respectively, of whom $54.9 \%$ were male, $93.4 \%$ were caucasian and $31.1 \%$ were diabetic. PETs were performed within the first $4.9 \pm 7.2$ months and the mean follow-up was $30.2 \pm 24.0$ months. During this period of follow-up, 52 (42.6\%) patients were transferred to long-term hemodialysis, 21 (17.2\%) received

\section{Table I}

Demographic, clinical and laboratory features of the study group

\begin{tabular}{|c|c|}
\hline Parameters & PD patients $(n=122)$ \\
\hline Age, years & $55.0 \pm 17.5$ \\
\hline Male gender, n (\%) & $67(54.9 \%)$ \\
\hline Caucasian, n (\%) & $114(93.4 \%)$ \\
\hline Diabetic, n (\%) & 38 (31.1\%) \\
\hline \multicolumn{2}{|l|}{ Renal diagnosis (\%) } \\
\hline Diabetic nephropathy & $24.6 \%$ \\
\hline Chronic glomerulonephritis & $17.2 \%$ \\
\hline Hypertensive nephrosclerosis & $9.0 \%$ \\
\hline Other / Unknown etiology & $49.2 \%$ \\
\hline $\mathrm{CCl}$ & $5.0 \pm 2.5$ \\
\hline Hemoglobin (g/dL) & $12.3 \pm 1.9$ \\
\hline Absolute neutrophil count $\left(\times 10^{9} / \mathrm{L}\right)$ & $5.6 \pm 2.5$ \\
\hline Absolute lymphocyte count (x10 1 L) & $1.8 \pm 2.3$ \\
\hline Absolute platelet count $\left(x 10^{9} / \mathrm{L}\right)$ & $269.6 \pm 86.5$ \\
\hline NLR & $3.99 \pm 2.6$ \\
\hline PLR & $195.5 \pm 101.7$ \\
\hline hs-CRP (mg/L) & $10.99 \pm 14.97$ \\
\hline $\mathrm{nGFR}\left(\mathrm{mL} / \mathrm{min} / 1.73 \mathrm{~m}^{2}\right)$ & $6.7 \pm 4.7$ \\
\hline Pre-albumin (mg/dL) & $39.3 \pm 113$ \\
\hline $\mathrm{D} / \mathrm{P}$ creatinine ratio & $0.6 \pm 0.12$ \\
\hline Creatinine clearance (L/week/1.73m²) & $101.3 \pm 43.3$ \\
\hline
\end{tabular}

Values are means $\pm S D$, unless specified otherwise. $C C I=$ Charlson comorbidity index; NLR = neutrophil-to-lymphocyte ratio; PLR = platelet-to-lymphocyte ratio; hs-CRP = high sensitivity C-reactive protein; $\mathrm{nGFR}=$ normalized glomerular filtration rate; $\mathrm{D} / \mathrm{P}$ creatinine ratio = dialysate to plasma creatinine ratio. kidney transplants and $3(2.5 \%)$ dropped out of the cohort. A total of $22(18 \%)$ patients died during the course of the study. Our population was dialyzed with a mean Kt/V 2.75 \pm 0.94 and a mean creatinine clearance $101 \pm 43.3 \mathrm{~L} /$ week $/ 1.73 \mathrm{~m}^{2}$, and the mean evaluated parameters were nGFR $6.7 \pm 4.7 \mathrm{ml} / \mathrm{min} / 1.73 \mathrm{~m} 2$, prealbumin $39.3 \pm 113 \mathrm{mg} / \mathrm{dL}$, hs-CRP $10.99 \pm 14.97 \mathrm{mg} / \mathrm{L}$, neutrophils $5.6 \pm 2.5 \times 10^{\wedge} 9 / \mathrm{L}$, lymphocytes $1.8 \pm 2.3 \times 10^{\wedge} 9 / \mathrm{L}$, and platelets $269.6 \pm 86.5 \times 10^{\wedge} 9 / \mathrm{L}$. Corresponding values for NLR and PLR were 3.99 \pm 2.6 and $195.5 \pm 101.7$, respectively. Patients' demographic, clinical and laboratory features are presented in Table I.

\section{Association between NLR, PLR and hs-CRP levels}

PLR was significantly and positively correlated with serum hs-CRP levels $(r=0.360, p<0.001)$. Similarly, NLR was also significantly and positively correlated with serum hs-CRP levels $(r=0.340, p<0.001)$.

\section{Mortality Predictability Comparisons of Selected Laboratory Variables}

On univariable Cox regression modeling, higher NLR, higher PLR, lower nGFR and higher $\mathrm{CCl}$ were significantly associated with risk of overall mortality. Separate models were performed for NLR and PLR associated factors. On both multivariable modeling, higher NLR and higher PLR, in conjunction with lower nGFR and higher CCl were significant independent predictors of poor survival, adjusting for prealbumin levels as a nutritional indicator (Table II and III).

\section{Table II}

Multivariable Cox regression analysis for NLR levels showing important predictors for overall mortality in PD patients.

\begin{tabular}{l|c|c}
\multirow{2}{*}{ Independent variables } & \multicolumn{2}{|c}{ All-cause mortality } \\
\cline { 2 - 3 } & Hazard ratio $(95 \% \mathrm{Cl})$ & P value \\
\hline nGFR & $0.706(0.554-0.900)$ & 0.005 \\
Pre-albumin & $1.023(0.974-1.074)$ & 0.365 \\
$\mathrm{CCl}$ & $1.650(1.174-2.320)$ & 0.004 \\
$\mathrm{NLR}$ (> mean vs. $\leq$ mean) & $1.662(1.117-2.472)$ & 0.012
\end{tabular}

$\mathrm{nGFR}=$ normalized glomerular filtration rate; $\mathrm{CCl}=$ Charlson comorbidity index; NLR = neutrophil-tolymphocyte ratio.

\section{Table III}

Multivariable Cox regression analysis for PLR levels showing important predictors for overall mortality in PD patients.

\begin{tabular}{c|c|c}
\multirow{2}{*}{ Independent variables } & \multicolumn{2}{|c}{ All-cause mortality } \\
\cline { 2 - 3 } & Hazard ratio $(95 \% \mathrm{Cl})$ & P value \\
\hline nGFR & $0.673(0.513-0.883)$ & 0.004 \\
\hline Pre-albumin & $1.023(0.977-1.071)$ & 0.332 \\
\hline CCI & $1.827(1.284-2.600)$ & 0.001 \\
\hline PLR (> mean vs. $\leq$ mean) & $1.010(1.004-1.015)$ & 0.001
\end{tabular}

$\mathrm{nGFR}=$ normalized glomerular filtration rate; $\mathrm{CCl}=$ Charlson comorbidity index; PLR = platelet-tolymphocyte ratio. 


\section{DISCUSSION}

Chronic inflammation is defined as a part of malnutrition-inflammation-atherosclerosis syndrome in patients with CKD and ESRD, leading to a considerable risk of total and cardiovascular death ${ }^{11}$. In this current study, $18.9 \%$ of patients died after a mean of follow-up time of 30 months, indicating a high risk of poor outcome in chronic dialysis patients. Similar outcomes have also been reported by other cohorts of PD patients, which underscores the need for additional parameters for risk stratification in PD patients ${ }^{12}$.

NLR and PLR, simple ratios obtained from a universally available low-cost complete blood count test routinely performed on admission, have been widely used as biomarkers of systemic inflammation ${ }^{13,14}$. Neutrophils may secrete a number of cytokines and activate other immune cells, thus promoting low-grade inflammation in the arterial wall ${ }^{15}$. On the other hand, studies demonstrated that activated platelets could incite leukocyte recruitment to the vessel wall and trigger the inflammation that can mainly be seen in the pathogenetic mechanism of atherosclerosis ${ }^{16,17}$. NLR was found to be significantly correlated with inflammatory markers, including CRP, pentraxin-3, tumor necrosis factor- $\alpha$ (TNF- $\alpha$ ) and interleukin- 6 (IL-6) in a population receiving renal replacement therapy ${ }^{18}$. PLR was also positively correlated with NLR ratio, IL- 6 and TNF- $\alpha$ levels. Additionally, ESRD patients with higher PLR values had higher levels of inflammation ${ }^{19}$. Our study findings were confirmatory to previous studies in regard to positive correlation between NLR and PLR with serum hs-CRP levels ${ }^{20,21}$.

Catabay C. et al. conducted a large-scale study including 108,548 hemodialysis patients which showed that high NLR value, but not PLR, provided additional benefit in predicting mortality ${ }^{10}$. Lu X. et al. enrolled 86 patients undergoing PD and suggest that high NLR is independently associated with arterial stiffness and predicts cardiovascular and all-cause mortality ${ }^{22}$. In our multivariate Cox regression analysis, NLR and PLR were found to be significant predictors of all-cause mortality in PD patients. Furthermore, this relationship was independent of various confounding factors, including $\mathrm{CCl}$, nGFR and pre-albumin. Our results also showed the excellent predictive value found for $\mathrm{CCl}$ and nGFR, in line with preceding evidence 23,24 . On the other hand, the lower predictive value found for pre-albumin may be related to the high standard deviation of our data.

Several limitations of this study should be considered. First, it was a small-sample observational, retrospective, single-institution study, which only enrolled 122 patients and thus may have weakened the power of some statistical analysis. Second, all the parameters were measured on a single occasion at the time of PET and did not take into account changes over time. Third, we did not look at medical conditions or treatments known to affect the CBC. Nevertheless, we collected data at the time of PET, eliminating possible confounding factors, such as infection, since this condition is a contraindication to perform this test, according to our unit protocol. Fourth, the current study is a prognostic rather than an etiologic study. Also, we only used serum hs-CRP as an inflammatory marker to correlate with NLR and PLR and, although statistically significant, these correlations are considered weak.

The findings of the present study indicated that baseline NLR and PLR measurements may provide a simple and inexpensive method for predicting mortality in chronic PD patients, when compared with other prognostic markers which may need specific equipment or reagent. Our analysis also suggests that NLR can predict mortality better than PLR in this population. Prospective studies are needed to investigate the potential prognostic impact of those markers.

\section{Authors' contribution}

Authorship of RCM. ECC, ATD and AMG provided clinical assistance, made a substantial contribution to the concept and design of the work and revised it critically for important intellectual content. IB provided clinical assistance. PLN revised it critically for important intellectual content. All authors approved the final manuscript.

Disclosure of potential conflicts of interest: none declared.

\section{References}

1. Azab B, Shah N, Akerman M, McGinn JT. Value of platelet/lymphocyte ratio as a predictor of allcause mortality after non-ST-elevation myocardial infarction. J Thromb Thrombolysis 2012; 34:326-334.

2. Yao CY, Liu XL, Tang Z. Prognostic role of neutrophil-lymphocyte ratio and platelet-lymphocyte ratio for hospital mortality in patients with AECOPD. Int J COPD. 2017; 12:2285-2290.

3. Amdur RL, Feldman HI, Gupta J, Yang W, Kanetsky P, Shlipak M, et al. Inflammation and progression of CKD: The CRIC study. Clin J Am Soc Nephrol. 2016; 11:1546-1156.

4. Neuen BL, Leather N, Greenwood AM, Gunnarsson R, Cho Y, Mantha ML. Neutrophil-lymphocyte ratio predicts cardiovascular and all-cause mortality in hemodialysis patients. Ren Fail. 2016; 38:70-76.

5. Sobrino-Márquez JM, Grande-Trillo A, Cantero-Pérez EM, Rangel-Sousa D, Lage-Galle E, AdsuarGómez A. Prognostic value of blood panel parameters in patients with dilated cardiomyopathy and advanced heart failure. Transplant Proc. 2018; 50:650-652.

6. Thio QCBS, Goudriaan WA, Janssen SJ, Paulino Pereira NR, Sciubba DM, Rosovksy RP, et al. Prognostic role of neutrophil-to-lymphocyte ratio and platelet-to-lymphocyte ratio in patients with bone metastases. Br J Cancer 2018; 119:737-743.

7. Okyay GU, Salih I, Er RE, Pa Ö, Derici Ü, Erten Y. Neutrophil to lymphocyte ratio in evaluation of inflammation in patients with chronic kidney disease 2013; 35:29-36.

8. Ahbap E, Sakaci T, Kara E, Sahutoglu T, Koc Y, Basturk T, et al. Neutrophil-to-lymphocyte ratio and platelet-to-lymphocyte ratio in evaluation of inflammation in end-stage renal disease. Clin Nephrol. 2016; 85:199-208.

9. Yoshitomi R, Nakayama M, Sakoh T, Fukui A, Katafuchi E, Seki M, et al. High neutrophil/lymphocyte ratio is associated with poor renal outcomes in Japanese patients with chronic kidney disease. Ren Fail. 2019; 41:238-243.

10. Catabay C, Obi Y, Streja E, Soohoo M, Park C, Rhee CM, et al. Lymphocyte cell ratios and mortality among incident hemodialysis patients. Am J Nephrol. 2017; 46:408-416.

11. Bal Z, Bal U, Okyay K, Yilmaz M, Balcioglu S, Turgay O, et al. Hematological parameters can predict the extent of coronary artery disease in patients with end-stage renal disease. Int Urol Nephrol. 2015; 47:1719-1725.

12. Unsal A, Koc Y, Basturk T, Sakaci T, Ahbap E, Sinangil A, et al. Clinical outcomes and mortality in peritoneal dialysis patients: a 10-year retrospective analysis in a single center. Clin Nephrol. 2013; 80:270-279.

13. Altunoren O, Akkus G, Sezal DT, Ciftcioglu M, Guzel FB, Isiktas S, et al. Does neutrophyl to lymphocyte ratio really predict chronic kidney disease progression? Int Urol Nephrol. 2019; 51:129137.

14. Akboga MK, Canpolat U, Yayla C, Ozcan F, Ozeke O, Topaloglu S, et al. Association of platelet to lymphocyte ratio with inflammation and severity of coronary atherosclerosis in patients with stable coronary artery disease. Angiology. 2016; 67:89-95.

15. Chen IC, Yu CC, Wu YH, Chao TH. Elevated neutrophil-to-lymphocyte ratio predicts intermediateterm outcomes in patients who have advanced chronic kidney disease with peripheral artery disease receiving percutaneous transluminal angioplasty. Acta Cardiol Sin. 2016; 32:532-541.

16. Uçar FM, Açar B, Gul M, Özeke Ö, Aydogdu S. The association between platelet/lymphocyte ratio and coronary artery disease severity in asymptomatic low ejection fraction patients. Korean Circ J. 2016; 46:821-826.

17. Langer HF, Gawaz M. Platelet-vessel wall interactions in atherosclerotic disease. Thromb Haemost. 2008; 99:480-486.

18. Turkmen K, Guney I, Yerlikaya FH, Tonbul HZ. The relationship between neutrophil-to-lymphocyte ratio and inflammation in end-stage renal disease patients. Ren Fail. 2012; 34:155-159.

19. Balta S, Demirkol S, Kucuk U. The platelet lymphocyte ratio may be useful inflammatory indicator in clinical practice. Hemodial Int. 2013; 17:668-669.

20. Yuan $Q$, Wang J, Peng Z, Zhou Q, Xiao X, Xie Y, et al. Neutrophil-to-lymphocyte ratio and incident end-stage renal disease in Chinese patients with chronic kidney disease: Results from the Chinese Cohort Study of Chronic Kidney Disease (C-STRIDE). J Transl Med. 2019; 17:1-8. 
21. Tatar E, Mirili C, Isikyakar T, Yaprak M, Guvercin G, Ozay E, et al. The association of neutrophil/ lymphocyte ratio and platelet/lymphocyte ratio with clinical outcomes in geriatric patients with stage 3-5 chronic kidney disease. Acta Clin Belgica Int J Clin Lab Med. 2016; 71:221-226.

22. Lu X, Wang S, Zhang G, Xiong R, Li H. High neutrophil-to-lymphocyte ratio is a significant predictor of cardiovascular and all-cause mortality in patients undergoing peritoneal dialysis. Kidney Blood Press Res. 2018; 43:490-499.

23. Fried L, Bernardini J, Piraino B. Charlson comorbidity index as a predictor of outcomes in incident peritoneal dialysis patients. Am J Kidney Dis. 2001; 37:337-342.

24. Shemin D, Bostom AG, Lambert C, Hill C, Kitsen J, Kliger AS. Residual renal fuction in a large cohort of peritoneal dialysis patients: change over time, impact on mortality and nutrition. Perit Dial Int. 2000; 20:439-444.

\section{Correspondence to:}

Roberto C. Marques, MD

Nephrology Department, Centro Hospitalar Universitário do Algarve, Faro - Portugal

E-mail: rcmarques@chalgarve.min-saude.pt 